\title{
The challenge of recruitment for neurotherapeutic clinical trials in spinal cord injury
}

\author{
Andrew R. Blight ${ }^{1} \cdot$ Jane Hsieh ${ }^{2} \cdot$ Armin Curt $^{3}$ - James W. Fawcett ${ }^{4} \cdot$ James D. Guest $^{5} \cdot$ Naomi Kleitman $^{6}$. \\ Shekar N. Kurpad ${ }^{7} \cdot$ Brian K. Kwon $^{8} \cdot$ Daniel P. Lammertse $^{9} \cdot$ Norbert Weidner $^{10} \cdot$ John D. Steeves ${ }^{11}$
}

Received: 15 January 2019 / Revised: 13 March 2019 / Accepted: 15 March 2019 / Published online: 8 April 2019

(C) International Spinal Cord Society 2019

\begin{abstract}
Study design Narrative review by individuals experienced in the recruitment of participants to neurotherapeutic clinical trials in spinal cord injury (SCI).

Objectives To identify key problems of recruitment and explore potential approaches to overcoming them.

Methods Published quantitative experience with recruitment of large-scale, experimental neurotherapeutic clinical studies targeting central nervous system and using primary outcome assessments validated for SCI over the last 3 decades was summarized. Based on this experience, potential approaches to improving recruitment were elicited from the authors.

Results The rate of recruitment has varied between studies, depending on protocol design and other factors, but particularly inclusion/exclusion criteria. The recruitment rate also ranged over an order of magnitude between individual centers in a given study. In older multicenter studies, average recruitment rate was approximately one person per study center per month. More recent trials experienced lower rates of recruitment and potential reasons for this trend were examined. The current roles and potential of various stakeholder organizations in addressing problems of recruitment were explored. In addition, recent developments in methodology may help reduce the number of subjects required for well-powered studies.

Conclusions Several approaches are emerging to improve clinical trial design, efficacy outcome measures, and quantifiable surrogate markers, all of which should reduce the number of participants required for adequate statistical power. There is a growing sense of cooperation between various stakeholders but more should be done to bring together consumer and provider groups to improve recruitment and the effectiveness and relevance of neurotherapeutic clinical trials.
\end{abstract}

$\triangle$ Andrew R. Blight

arborlight@gmail.com

Acorda Therapeutics, Inc., Ardsley, NY, USA

2 Wings for Life, Salzburg, Austria

3 Spinal Cord Injury Center, University Hospital Balgrist, Zurich, Switzerland

4 Cambridge University Centre for Brain Repair, Cambridge, UK

5 Department of Neurosurgery, University of Miami, Miami, FL, USA

6 Craig H. Neilsen Foundation, Encino, CA, USA

7 Department of Neurosurgery, Medical College of Wisconsin, Milwaukee, Wisconsin, USA

8 Vancouver Spine Surgery Institute and ICORD, University of British Columbia, Vancouver, Canada

9 Craig Hospital, Englewood, CO, USA

10 Spinal Cord Injury Center, Heidelberg University Hospital, Heidelberg, Germany

11 ICORD, University of British Columbia, Vancouver, Canada

\section{Introduction}

In 2007, an international panel, supported by multiple foundations, under the banner International Campaign for Cures of Spinal Cord Injury Paralysis (ICCP) published a set of papers reviewing spinal cord injury (SCI) clinical trial methodology, and providing recommendations for future studies [1-4]. Almost a decade later, several foundations (see Funders) sponsored another meeting to address both new and persistent barriers to translational research success in SCI. One result of this coalition was establishment of a new initiative, the Spinal Trials Understanding, Design, and Implementation (STUDI) group, to consider and report on ways to improve participant recruitment, study protocol design and trial outcome measures, including electrophysiology, neuro-imaging, and chemical biomarkers. These reports are to be presented in a series of papers, including the current work, which specifically addresses recruitment issues and strategies for SCI clinical trials. 
The particular focus of these efforts are experimental neurotherapeutic approaches to SCI, studies looking at interventions that specifically address the central nervous system (CNS) damage in SCI, usually through neuroprotection and repair strategies. This review does not aim to provide a comprehensive and systematic review of all randomized controlled trials (RCTs) conducted worldwide in the context of SCI. Indeed, the recruitment issues in different types of SCI study, those directed to other aspects of acute and chronic SCI care, such as surgical stabilization and rehabilitation techniques, are likely to be distinct. We do not consider the current paper or the set of papers of which it forms a part to be the end of a discussion of trial design and execution, but a seed from which to build.

Clinical development of new therapies for SCI involves many challenges. Recruitment of adequate numbers of appropriate participants and qualified study centers for large-scale pivotal trials is prominent among them. Recruitment was not an insurmountable problem in past decades. A number of trials, involving hundreds of individuals with SCI, were completed within reasonable timeframes. More recently, recruitment has proven difficult for several programs, leading to delay or even abandonment of some trials. An examination of whether this reflects real change appears warranted.

Some problems of recruitment are universal and others are specific to or modified by the condition of interest. In the case of neurotherapeutic approaches to SCI, specific issues vary across acute, subacute and chronic injury and between different types of planned intervention, from neuromodulation techniques, through drugs and biologics to biomaterials and cell or tissue transplantation. Our interest in assembling this review was to focus on concerns specific to SCI, including ways to address them.

The most fundamental limitations on recruitment lie in the demographics of SCI and the characteristics of the healthcare systems. These include the number of individuals injured, the number of trial centers available, and the proportion of the population to which those centers have access within the time window available for treatment. Precise information on these parameters is not readily available, though approximations can be made for some regions of the world $[5,6]$. It is thus possible to estimate the number of advanced development programs that can feasibly run simultaneously. Analyses of this kind were recently performed for Canadian sites [7, 8].

Recruitment capacity is further limited by the fact that not all centers involved with SCI care are both capable of and interested in participating in trials. In the United States (USA), there has been a significant increase in the number of Level 2 trauma centers treating acute SCI. These centers often have lower case numbers than Level 1 centers and may lack research infrastructure. Lower numbers may also affect individual outcomes [9]. This decentralization represents a potentially addressable challenge. Enhanced coordination among the constituencies involved in funding, performing, and regulating SCI care and research should be encouraged to increase integration to enhance participation of appropriate individuals [10].

In addition, many of the details of study design and management restrict the population eligible to participate in a trial. Sometimes this is unavoidable to ensure safety and increase the likelihood of meaningful results. Furthermore, not all individuals are willing to participate in clinical research. Factors affecting the likelihood of volunteering include age [11], gender and education [12]. For acute therapeutic studies, injury severity is a key selection factor, and polytrauma (e.g., head injury) may preclude enrollment.

\section{Historical background}

Early stages of clinical development can usually be performed with limited concern for recruitment issues and such studies are often appropriate for completion in one or a few centers. Phase 1 and 2 trials, which provide insight into the safety and potential for efficacy of an intervention, generally require 10-100 subjects, skewed towards the lower number under the pressure of cost $[13,14]$. Some Phase 2 trials are undertaken with the (usually unrealistic) hope of providing strong evidence of efficacy, so they might ultimately be considered as equivalent to a Phase 3 study. In such cases, recruitment may include 100 or more people, increasing the time and cost to complete recruitment. Pivotal Phase 3 studies, required for credible demonstration of efficacy and safety, usually require at least a few hundred individuals, based on the outcome measures available in SCI and using more conservative powering assumptions. Power is calculated from expected (usually relatively small) effects occurring against a background of spontaneous variability in outcome measures, to the extent that these parameters are known or can be reasonably estimated. Regulatory agencies are not usually concerned whether a particular trial has been labeled as Phase 2 or Phase 3, only whether it meets criteria for being "adequate and well-controlled", allowing meaningful conclusions to be drawn.

Only a few, fully powered, randomized, double-blind, Phase 3 studies of experimental neurotherapeutic approaches have been completed and published in SCI. Most of those in acute SCI were performed in the 1980s and 1990s and those in chronic SCI mainly in 2000-2004. In addition to these studies, a much larger number of trials, including RCTs have been performed that were focused on other aspects of SCI care and management. Those include extensive studies of different rehabilitation techniques and 
symptomatic treatments that do not directly address the CNS. No large, prospective neurotherapeutic trials have been completed and published in the most recent decade. Some smaller, exploratory studies have been published and several larger scale studies are recorded as ongoing in current databases.

\section{Acute SCI-NASCIS}

The first large-scale study was organized by the National Acute Spinal Cord Injury Study group (NASCIS), a coalition of clinicians and researchers at trauma centers in the USA. This study was designed to compare high and low doses of methylprednisolone sodium succinate. Glucocorticoid drugs were already widely used to treat SCI (as well as a large range of other conditions) without clear evidence of efficacy or relative safety. This meant investigators felt ethically unable to perform the study with a placebo control. Between February 1979 and November 1981, 330 people were randomized across 9 hospitals, 6 of which were specialized SCI centers. The recruitment at individual centers ranged from 10 to 80 . The key recruitment criteria included admission within $48 \mathrm{~h}$ of injury and diagnosis of acute SCI by an attending neurosurgeon, including any loss of sensory or motor function below the level of injury [15].

Following the negative outcome of this study, and on the basis of new preclinical data, the investigators then performed a second study (NASCIS 2), comparing three treatments groups: very high dose methylprednisolone for $24 \mathrm{~h}$, naloxone and placebo. Between May 1985 and December 1988, this study randomized 487 people across 10 centers in the USA, the recruitment at individual centers ranging from 11 to 103 . The average time from injury to first treatment was between 8 and $9 \mathrm{~h}$ across the treatment groups. Recruitment criteria were similar to NASCIS 1 except that randomization to a treatment group was required within $12 \mathrm{~h}$ of injury [16, 17].

Following what was interpreted in NASCIS 2 as a beneficial effect of methylprednisolone for acute SCI patients treated within $8 \mathrm{~h}$ of injury, a third trial (NASCIS 3) was performed to compare a $24 \mathrm{~h}$ regimen of methylprednisolone to a $48 \mathrm{~h}$ regimen, and a $48 \mathrm{~h}$ regimen of tirilazad mesylate. Between December 1991 and September 1995, a total of 499 people were randomized, out of 788 screened ("registered") across 16 centers in the USA and Canada. The recruitment at individual centers ranged from 2 to 76 . Again, the key recruitment criteria were similar except that the required time from injury to treatment was reduced to $8 \mathrm{~h} \mathrm{[18].} \mathrm{The} \mathrm{results} \mathrm{of} \mathrm{NASCIS} 2$ and 3 continue to be debated, but the apparent effect size was small and substantial adverse events occurred.

\section{GM-1 ganglioside}

A small, Phase 2, single-center study of GM-1 ganglioside was performed between January 1986 and May 1987. All of the 351 individuals with SCI admitted to a single trauma center during that period were screened for inclusion in the trial, and 37 were randomized to treatment with GM-1 ganglioside or placebo. The key inclusion criteria included a major motor deficit in the upper or lower extremities. The great majority of exclusions (210 of 214) were for lack of sufficient neurological deficits [19].

Based on the suggestion of efficacy in this study, a large Phase 3 study of GM-1 was conducted, which randomized 797 people across 28 neurotrauma centers over 5 years from April 1992 to January 1997. Recruitment at individual centers ranged up to 76 . In total, 3165 individuals were screened and 2368 excluded before randomization. The key recruitment criteria included at least one lower extremity with an International Standards for the Classification of Spinal Cord Injury (ISNCSCI) motor score less than 15 out of a potential 25 . The published reporting of this study was unique in the field in its completeness and the experience with recruitment is described in excellent detail. As in the earlier trial, the largest category of exclusion was lack of sufficient neurological deficit $[20,21]$. The overall study result was negative with regard to efficacy.

\section{Gacyclidine}

A large multi-center "Phase 2" study of gacyclidine, a glutamate antagonist, in acute SCI was performed in France, recruiting 280 people between February 1995 and November 1998. The study involved "126 emergency units, 31 surgical and intensive care units, and 29 rehabilitation units" across France. The key recruitment criteria included acute SCI with a maximum motor score of 15 for the most severely injured lower limb. Treatment had to be initiated within $2 \mathrm{~h}$ after injury so the study required community assent [22]. This approach to hyperacute treatment is not often possible. The treatment did not show significant efficacy.

\section{Subacute SCl-autologous macrophages}

An RCT of transplanted autologous "activated" macrophages was undertaken, but terminated for financial reasons before recruitment was completed. This study is of interest primarily because the recruitment experience was thoroughly documented, with a discussion of the "funnel effect" [23]. This term is an analogy for the drastic reduction in available participant numbers produced by each stage of selection from initial screening to final randomization. The 
effect, which includes among other matters geographic constraints, consent process, and study requirements, was exacerbated in this study by stringent recruitment criteria, and the requirement that the intervention was delivered within a 14 day window from the time of injury.

This time window meant that the study did not fall into the usual categories of acute or chronic treatment, though there is no general agreement on times that define "acute" and "chronic" in SCI. This study illustrates the particular difficulty involved in the more complex trials required for cell therapies with surgical intervention. A total of 1816 people were prescreened, 75 evaluated in more detail, and 50 randomized between October 2003 and May 2006. Individuals with a single lesion were required to be motor complete at a level of C5 or below [23]. The treatment time window allowed recruitment of participants admitted and stabilized at nonstudy centers who could be transferred at the sponsor's expense to study centers, thus enabling a very large "catchment" area. The trial did not provide evidence of clinical benefit.

\section{Chronic SCI}

\section{Tizanidine}

A successful Phase 3 study of tizanidine hydrochloride for treatment of spasticity in chronic SCI was performed in the early 1990s, randomizing 124 individuals across 14 centers in a double-blind controlled trial [24]. Unfortunately, the timing of recruitment was not reported so it is not possible to derive information on recruitment rate and therefore the study is not considered further for this current discussion.

\section{4-Aminopyridine}

A series of three large studies in chronic SCI, were carried out with 4-aminopyridine (fampridine or dalfampridine). The first was a Phase 2 study directed at a theoretically broader potential for improving conduction in demyelinated central nerve fibers. It involved 11 rehabilitation research centers across North America, randomizing 91 people between June and November 2000. A total of 129 people were assessed for eligibility [25]. The results suggested a benefit on moderate to severe spasticity.

This was followed by two identical, parallel Phase 3 studies, involving 78 rehabilitation centers in North America, more narrowly addressing spasticity effects, though including other secondary measures of neurological function. Across the two trials, a total of 417 people were enrolled and randomized between June 2002 and August 2003. The target population was individuals with chronic, incomplete SCI with spasticity, whose injury occurred at least 18 months prior to screening, and whose neurological status had been stable for at least 6 months [26]. There was no significant effect on spasticity in these trials.

A large Phase 2 study was performed in 2004-2005 to study the effects of another ion channel drug, HP-184, in chronic SCI (ClinicalTrials.gov Identifier: NCT00093275). This also failed to show efficacy and the details of the study were not published. Other trials of potential neurotherapeutic interventions are known to the authors to have been performed around the world that were not published in sufficient detail to provide information on recruitment. The historical analysis we provide has an apparent bias to studies carried out in North America. This reflects in part the fact that most studies of this kind have been based in the USA and Canada for a variety of reasons, including demographic and economic. However, the failure to publish clinical trial information, particularly in negative studies, has exaggerated the appearance of North American dominance in the field.

\section{Overall historical experience with large neurotherapeutic SCl studies}

A summary of the historical rates of recruitment in large SCI studies of experimental neurotherapeutic interventions is provided in Table 1. The calculated recruitment rates at the site level are approximate because not all the details of individual trial site involvement is known, so estimates of the number of people recruited per site per month were derived by assuming all sites were recruiting throughout the study period. This is often not the case, which means actual per month rates per site may have been slightly higher. The values given for number of people screened is unreliable, since ways of defining and performing screening differ between trials, including different degrees of prescreening. An extreme case is the first GM-1 ganglioside study [19], which screened everyone with SCI presenting at the acute trauma center over 16 months. The entire cohort reported as being "evaluated" for inclusion was an order of magnitude greater than the number actually randomized, even though recruitment criteria were fairly broad. Thus, it is difficult to reliably characterize the extent and severity of the "funnel effect" given that recruitment details are often incompletely reported.

A few observations are striking in Table 1. First, over 25 years, average recruitment rates in acute and chronic SCI trials were not widely different. Recruitment rates tended to trend downward over time, but it is not clear if this is related to changes in research infrastructure, to the increasing complexity of trial design and recruitment criteria, or to a variety of factors. Excluding the gacyclidine study [22], where it is unclear how many centers contributed to 
Table 1 Recruitment rates in historical large neurotherapeutic SCI studies

\begin{tabular}{lllllll}
\hline Study & $\begin{array}{l}\text { Subjects } \\
\text { "Screened" }\end{array}$ & Randomized & & Sites & Months & $\begin{array}{l}\text { Subjects/site/month } \\
\text { Mean }\end{array}$ \\
\hline NASCIS 1 [14] & & 330 & 9 & 33 & 1.11 & Range \\
NASCIS 2 [15] & & 487 & 10 & 43 & 1.13 & $0.30-2.42$ \\
NASCIS 3 [17] & 788 & 499 & 16 & 45 & 0.69 & $0.25-2.40$ \\
GM1 Ph2 [18] & 351 & 37 & 1 & 16 & 2.31 & $0.04-1.69$ \\
GM1 Ph3 [19] & 3165 & 797 & 28 & 57 & 0.50 & NA \\
Gacyclidine [21] & & 280 & $157(?)$ & 45 & 0.04 & $?-1.33$ \\
Macrophage [22] & 1816 & 50 & 6 & 30 & 0.28 & $?$ \\
4-AP Ph2 [24] & & 91 & 11 & 6 & 1.38 & $0.00-0.60$ \\
4-AP Ph3 [25] & & 417 & 78 & 14 & 0.38 & $0.50-2.17$ \\
Acute & & & & 1.15 & $0.07-1.21$ \\
Chronic & & & & & 0.88 & $0.04-2.42$ \\
\hline
\end{tabular}

? unpublished, NA not applicable, acute mean of first 5 studies only, chronic mean of last 2 trials only. The last two columns show the average number of subjects per site per month recruited into a given study and the range of recruitment rates throughout the study for individual centers within the study recruitment, and the single center Phase 2 GM1 study [19], the mean recruitment rate varies from 0.27 to 1.38 people per site per month. Restricted to large acute-SCI studies, the numbers are even closer, at $0.50-1.13$. At least one of the largest ongoing acute SCI trials [27] is falling below this historical range, even with international collaboration (USA, Canada, and Australia). Again, many factors may contribute to the decrease in rate of recruitment. One possibility is that the failure to show efficacy in large trials of previous decades has decreased investigator and/or participant optimism regarding the likely outcome of new studies.

The second observation is that the average rate of recruitment to Phase 2 studies was higher than that to Phase 3 studies, though it was not higher than the highest recruitment rates achieved at the most effective centers in Phase 3 trials. The third point, related to this, is that the range of recruitment rates across centers in a given multicenter trial is quite high, usually an order of magnitude. However, the highest individual center recruitment rate was fairly consistent across trials, ranging from 1.21 to 2.42 people per center per month, and this was consistent with the rate of 2.31 seen in the single center GM1 study [19], which may be indicative of the maximum possible recruitment at a single, large trauma center.

\section{Major trial characteristics that affect recruitment}

Large-scale studies performed in past decades tended to be relatively simple in design and often had only moderately restrictive inclusion and exclusion requirements. Those factors may have been important contributors to their timely completion. The investment of time and money required by a clinical trial tends to encourage attempts to acquire as much information as possible. This is motivated in part as insurance against having picked the wrong primary measure, but also with the laudable scientific desire to learn as much as possible. However, the addition of multiple secondary goals to a protocol may work against recruitment and retention by making the study less attractive to centers and individuals considering participation, and make it more difficult to achieve the primary goal. An excessively complex study may run out of time or money before it is fully enrolled, or the burden on research staff and participants may lead to collection of incomplete or unreliable data. Any study, even a successful one, will usually leave us with more questions than answers. The primary goal should be to ensure that we answer the one primary question definitively and efficiently.

Many factors affect recruitment and the severity of the "funnel effect", the gradual reduction of the number of appropriate candidates from the population, caused by deliberate or collateral selection pressures. The first selection factor is usually the decision whether the trial is directed to treatment of acute or chronic SCI. There are several reasons to think that chronic injury studies should be easier to recruit. There are many more individuals at any given time living with chronic injury and they have passed through the more complex acute care stage. If we think of recruitment occurring over 2 or 3 years, then (based on average age of injury and life expectancy of individuals with SCI) there should be at least an order of magnitude more people available for chronic studies over the course of 1-2 years. Secondly, if we are considering a "hyperacute" injury, as is the case with most experimental approaches to neuroprotection, then the problem of accessing those individuals at the available study centers in the available few hours after injury will significantly reduce the available pool of participants. Thirdly, the number of people required may 
be lower for chronic injuries where therapeutic effects can be measured against a relatively stable neurological baseline, whereas in the acute setting treatment effects are likely to be more difficult to detect among the 'noise' created by spontaneous neurologic recovery, other injuries, and medical complications.

However, there are problems of recruitment with chronic injury as well. While individuals and their families in the acute stages of injury may urgently seek experimental treatments to improve sensorimotor recovery, chronically injured individuals may have different priorities [28]. Their main interest during clinical follow-up may be more focused on the secondary conditions profoundly affecting quality of life, such as bowel/bladder/sexual dysfunction, spasticity, neuropathic pain, pressure injuries, and autonomic dysreflexia. Such individuals may be reluctant to jeopardize neurologic gains acquired during rehabilitation and the precious function they currently have in order to participate in an experimental trial that entails inherent risk [29-31].

Many individuals otherwise suitable for participation in a study will not be living within commuting distance of a major research center or have the resources to travel to participate in a study involving numerous visits. In addition, many experimental approaches to chronic SCI are likely to be relatively selective in terms of the type and severity of injury that would be appropriate to address.

As an example, the Stem Cells Inc. Phase 1 clinical trial recruiting people with thoracic injuries (ASIA Impairment Scale (AIS) A and B) from 4 to 24 months postinjury considered 1550 candidates worldwide. Of those, only 50 candidates were invited to be assessed for eligibility and 12 were deemed appropriate for enrollment (Curt A, Casha S, Fehlings M, personal communication). In the Phase 2 cervical SCI trial of HuCNS-SC cell transplantation, 31 of 64 subjects (AIS A and B) passed screening [32], but the total number of participant queries is unknown (likely in the hundreds).

The type of intervention under consideration will affect recruitment. This may range from approved oral drugs, through novel drugs or biologics, devices, cell transplants, surgery, or therapies that combine more than one of these. Rehabilitation techniques, which may be a topic of study in themselves, may also form part of a "combination therapy". As the intervention becomes more complex, this usually increases the selection pressure on candidates for participation and decreases the likelihood of obtaining consent and retaining participation throughout the trial. There is also likely to be a related greater cost of treating each person, increasing pressure to recruit "ideal" candidates.

The degree of surgical invasiveness of an intervention, the potential reversibility of its effects, and the extent of prior knowledge of its safety will all affect the readiness with which centers and candidates volunteer and provide consent. The cumulative burden of follow-up measures (e.g., cerebrospinal fluid samples, magnetic resonance imaging, testing of sensory and motor function, duration and frequency of visits) will all affect the willingness of potential participants to commit to a study.

\section{Additional trial details that limit recruitment}

With regard to estimating expected funnel effects, there are many other factors to bear in mind. Some exclusion criteria are standard for clinical trials in any condition. Among these are steps to protect the most vulnerable from experimental interventions of incompletely known safety, including age restrictions, exclusion of pregnant women, and individuals with concomitant conditions or medications that might interact with the treatment or confound outcome measures. There are also considerations to avoid collection of incomplete or unreliable data, excluding people with questionable ability to complete study procedures, based on such factors as a history of substance abuse.

Other likely exclusion criteria are more specific to SCI, such as the avoidance of the potentially different neuropathological mechanisms introduced by penetrating injuries, gunshot or multilevel injuries, and the presence of concomitant conditions such as brain trauma or major organ damage, and complications such as pneumonia and respiratory failure, which are frequently encountered in acute and subacute studies. Individuals requiring mechanical ventilation or tracheostomy will often be excluded from trials for a number of reasons including safety concerns and increased difficulty of consent and neurological examination. Drug and alcohol involvement in traumatic injury may also prevent recruitment to acute studies. Most protocols will require conscious and responsive individuals for a baseline neurological assessment and for consent to be obtained.

\section{The problem of retention}

The longer the study duration, the more difficult it is to retain every recruited individual and obtain the full complement of follow-up assessments. In some cases, retention is as much a concern as recruitment for acquiring sufficient data. Given the natural history of neurological recovery from SCI, the earliest long-term assessment liable to give a good estimate of the ultimate limit of recovery is about 6 months after injury, though most studies will also include a 1 year follow up, closer to the asymptote for neurological recovery [1]. Distance from the individual's home to the study center is a factor in retention. Studies frequently need 
Table 2 Planned recruitment in SCI trials currently registered with www.clinicaltrials.gov (via www.scope-sci.org listing)

\begin{tabular}{lllllll}
\hline Neuro-therapeutic trials & $\begin{array}{l}\text { Number of Trials } \\
\text { (acute/chronic/sub/NS) }\end{array}$ & $\begin{array}{l}\text { Planned } \\
\text { recruitment }\end{array}$ & Acute & Chronic & Subacute $^{\mathrm{a}}$ & NS \\
North America & $20(8 / 11 / 1 / 0)$ & $1623^{\mathrm{b}}$ & $928^{\mathrm{b}}$ & 570 & 125 & - \\
Europe & $4(2 / 2 / 0 / 0)$ & 198 & 146 & 52 & - & - \\
Rest of World & $12(2 / 8 / 1 / 1)$ & 712 & 90 & 392 & 130 & 100 \\
Other trials & & & & & & \\
& & Planned & Acute & Chronic & Subacute & NS \\
North America & $64(2 / 55 / 1 / 6)$ & 2624 & 140 & 2474 & 10 & 138 \\
Europe & $22(4 / 16 / 1 / 1)$ & 583 & 278 & 305 & 20 & 50 \\
Rest of World & $7(1 / 5 / 0 / 1)$ & 409 & 200 & $192^{\mathrm{a}}$ & 0 & 17 \\
\hline
\end{tabular}

$N S$ type of injury not specified or trial includes both acute and chronic injuries.

${ }^{a}$ Listing purely subacute trials, several studies include subacute SCI with predominantly acute or chronic cases, but without specifying numbers and with various temporal definitions of subacute

${ }^{\mathrm{b}}$ One acute study with planned enrollment of 351 plans recruitment in Australasia in addition to North America to include financial support for follow-up travel and accommodation and in some cases it may be possible to send an assessor to the individual's home.

Several other factors contribute to retention failure, but the burden of numerous study assessments can be a disincentive. It is helpful to make the follow-up atmosphere as welcoming as possible and to address medical, social and psychological issues during visits, to increase the perceived value of follow up and to help participants know their contributions are essential and appreciated.

\section{Current clinical trials in SCI}

Ongoing clinical studies related to SCI have been summarized and tabulated over recent years by SCOPE (Spinal Cord Outcomes Partnership Endeavor) and published on its website (http://scope-sci.org/trials/). This tabulation curates information from the U.S. National Institutes of Health website (https://clinicaltrials.gov/) and presents enrolling (or soon to be enrolling) trials from around the world that involve neurotherapeutic interventions, particularly those studies where the sponsors have an interest in eventually addressing regulatory review in the USA. In a recent iteration of these tables (December 2018) trials were broken down into two sets: 85 trials of rehabilitation or "technological intervention" and 42 trials of drug, cell, or surgical interventions. Some of these trials involve combinations of approaches, which can make classification difficult, and although this dichotomy separates to a large extent experimental neurotherapeutic approaches from other aspects of SCI research, there are a few exceptions, as in the case of three studies of peripheral nerve transfer and three studies of timing of decompression surgery which appear in the table of "drug, cell or surgical interventions", but in other respects fit better with the "rehabilitation or technological" category.

Table 2 is extracted from the SCOPE tables and summarizes the planned enrollment of participants in two slightly modified categories: broadly neurotherapeutic trials (excluding the six trials mentioned above) and studies addressing other aspects of SCI rehabilitation and management (including those six studies with all of the "rehabilitation and technological" studies. For the present, studies of CNS stimulation (electrical or magnetic) have been included with this latter category. As more is learned about the mechanism and durability of the effects of such interventions, that categorization may be re-examined.

Some of the trials in the neurotherapeutic category are large RCTs that would compete directly for participants, but all these studies represent some level of competition and it is useful to consider their extent. A full review of international activity would require similar analyses of other databases, such as the European (www.clinicaltrialsregister. eu) and Australasian (www.anzctr.org.au.) sites.

Given the incidence and prevalence on SCI around the world [5, 6] recruitment of more than 6000 people worldwide should not be difficult, given a sufficiently wide geographical distribution of sites and sufficient time for recruitment. However, specifically with regard to those trials planned in the USA, and given the constraints of the "funnel effect" described above, severe competition for recruitment of both qualifying participants and research centers is to be expected, especially when those individuals with milder severity of SCI (e.g., AIS D) are often excluded from acute neurotherapeutic trials due to significant 
spontaneous functional recovery within this category. From Table 2, ongoing studies in North America plan to recruit almost 3000 participants with chronic injury and over 1000 with acute or subacute injury.

\section{The European perspective}

Europe has been relatively underrepresented in SCI clinical trials. The European Union has 508 million inhabitants (https://europa.eu/european-union/about-eu/figures/living en) compared to a total of 365 million people living in the USA and Canada. The incidence of traumatic SCI is similar, although difficult to compare due to the wide variation in estimates (20.7-83.0 per million inhabitants a year in North America versus 8.0-130.6 in Europe) [6, 33]. In contrast to the increasing number of clinical trials conducted in North America, relatively low numbers are registered across Europe. Thus, increased access to European centers would help to overcome the potential bottleneck in North America.

Excellent SCI research networks exist in Europe. European Multicenter Study about Spinal Cord Injury (EMSCI) is a research network of 21 active SCI centers in Europe and a coherent platform of clinical sites, which gathered expertize required for clinical trials over many years. A total of 300 professionals have been trained since 2006 in centralized courses to reliably perform the ISNCSCI and the Spinal Cord Independence Measure (SCIM) assessments, with close monitoring of individual performance [34]. This served as a basis of a Phase 1 clinical trial investigating the feasibility and safety of intrathecal administration of an antiNogo antibody in people with traumatic paraplegia and tetraplegia [35]. EMSCI sites continue to assess people with acute SCI to further characterize the pattern of natural recovery, and a Phase 2 study of anti-Nogo administration in SCI patients is planned.

However, there are distinct differences in SCI care between the USA and Europe. Unlike many U.S. centers, European, and Canadian rehabilitation centers treat people living with SCI as inpatients for several months after injury. It will be important to track differences in the level and duration of care after SCI as these may influence trial outcomes.

\section{The International Outlook}

Although the majority of large, randomized SCI neurotherapeutic studies published to date have focused on North America or Europe, neurotrauma is an international problem that has a higher incidence in a number of other parts of the world. An example of what is possible is provided by the MRC CRASH trial that was conducted to test steroids in head injury in 14 countries enrolling 10,000 subjects [36]. Such trials have not been completed in SCI. There is considerable potential for expanding clinical trial activity in countries with high population, high incidence of SCI and growing economic presence, such as China and India. While well organized trials are possible in these areas and some have been published [37] these have not yet made full use of the remarkable numerical potential presented by populations in Asia.

Despite the relatively small population to work with, the SCI research community in Australasia is extremely active, with a large number of SCI studies registered on its online database (www.anzctr.org.au). Most of these studies relate to aspects of care and management rather than late stage testing of neurotherapeutics. Randomized trials of therapeutics like Riluzole [38], to which Australian centers have contributed are also listed on www.clinicaltrials.gov. This includes completed trials that have not been published, such as earlier stage Anti-Mag, and Nerispirdine (HP-184) studies.

\section{The future of clinical trials in $\mathrm{SCl}$}

An increasing number of registered clinical studies raises the question of how many concurrent trials can be run successfully, and what might be done to increase that number so promising approaches do not languish untested. The question of what constitutes sufficient preclinical evidence to justify clinical development is an important and intimately associated problem [39-41] outside the scope of the current paper. The goal of this review has been to clarify the nature and extent of recruitment issues, but it was already clear that ongoing studies are encountering significant problems [42]. This is particularly concerning for corporate-sponsored programs, because shareholders require predictable timelines that are as short as possible. Government agencies and nongovernmental organizations (NGOs) may have similar concerns for their financial support.

Some current efforts to address recruitment issues are aimed at methodological improvements to reduce the numbers needed to recruit or to increase the proportion of injured individuals that can be recruited. Although more diverse populations would be expected to increase the variability of outcomes, improved understanding of the natural history of recovery may help to decrease numbers needed and increase breadth of recruitment.

\section{Prediction models for inclusive protocols}

Understanding the timing and extent of neurological and functional changes in SCI, is important for rational trial 
design. Appropriate powering of studies requires knowledge of statistical parameters of the chosen outcome measure, such as the minimum detectable difference, the minimal clinically important difference [43] and the variability of outcome relative to baseline assessments obtained.

A recent effort to improve understanding of natural recovery was based on analysis of the existing databases of the EMSCI trial network. This program [44-46] targeted advanced patient stratification using prediction models based on unbiased recursive partitioning (URP) of the targeted primary endpoint, with the goals of (a) prospectively addressing ceiling effects and exclusion of individuals unlikely to benefit from the intervention, i.e., those likely to achieve full recovery without treatment, (b) defining baseline clinical conditions with very poor outcomes, to avoid floor effects where treatment is unlikely to produce meaningful improvement in outcome, and (c) enabling enrollment of individuals with widely different levels or severities of SCI in a single trial, thereby avoiding the need for sequential trials.

\section{Improving outcome measures}

Another major methodological improvement, emerging from the analysis of the EMSCI database, was the development of a new scale of functional recovery, SCAR or Spinal Cord Ability Ruler [47]. This scale, which reflects the natural history of injury severity and the recovery of volitional movement, was designed with improved measurement characteristics, based on Rasch analysis, to increase the efficiency of clinical trials. Most of the existing functional scales widely used as outcome measures, such as the ISNCSCI and SCIM [48], constitute excellent means of classifying functional status but are not well suited for measuring effects of treatment on outcome. SCAR provides a linear, interval-scaled measure that has been specifically designed to measure the degree of volitional motor control along one axis of recovery. Furthermore, by combining aspects of the widely utilized ISNCSCI and SCIM assessments, a person with SCI can be tracked with SCAR longterm from the earliest acute evaluation. SCAR shows promise for increasing the ability to detect treatment effects using smaller numbers of individuals, though it needs further validation with prospective studies that confirm its theoretical advantages.

\section{Infrastructure and recruitment}

Acute-SCI studies with a limited time window for efficacy require the recruiting center to establish an "infrastructure" of on-call suitably trained research personnel, who are skilled in neurological and functional assessments and are available on an urgent basis to evaluate and consent potential participants. These personnel must cope with competing clinical demands. The challenge of establishing such a stable infrastructure is often underestimated by researchers and sponsors. Acknowledgment that clinical care and research require different resources is important when planning trials. Recruitment has been negatively impacted when infrastructure (e.g., dedicated study coordinator and local recruitment coordinators) is limiting [49].

As an example, there is widespread acceptance in the field that standardization of the ISNCSCI examination is critical and most trials include formal training for research staff members who will be performing assessments within the trial. The precision of the ISNSCI assessment (and ultimately the interpretation of therapeutic effect) will be improved by using dedicated clinical research personnel who are formally trained, can focus and devote the time required to do careful examinations, and are available for the follow-up examinations. In addition, there must be a system of communication to alert investigators of potential candidates for recruitment and they must be adequately empowered to access them during a stressful period of health care management. In a review of over 400 acute SCI patients admitted to a regional SCI referral center, the basic considerations of sedation, intoxication, and multiple injuries would have excluded approximately a third of all patients as "un-examinable", without considering any other clinical trial inclusion criteria [7].

Coordination of research centers should be a core goal, equivalent to the kinds of collaboration that were possible for the NASCIS effort in acute SCI and the Model Systems Centers, which played an important role in facilitating and acting as the nucleus for the multicenter studies of 4aminopyridine. In both cases, government funding played a role in forming the inter-institutional collaboration prior to development of Phase 3 protocols. Formation of these coalitions was fostered by the clinical academic culture of the time, where both the desire to do something about SCI and the availability of professional time set aside for research contributed to the effort and to the human drive that mitigated the effects of limited funding. That culture, at least within the USA, has seen marked negative changes in the 40 years since NASCIS was organized, making the task more difficult today, despite technological advances facilitating communication and coordination. Finally, industry involvement plays an important role, and this comes with its own cultural challenges, where large corporations often see SCI and neurological disorders in general as clinical targets where the risks and difficulties outweigh the potential rewards, especially given their relatively low incidence. Smaller corporations, which may be more committed to tackling difficult problems and innovative solutions, often lack the resources to push through development to completion, no matter how enthusiastic they may be. 
A reasonable goal for those interested in fostering SCI clinical development, including government and nonprofit groups, would be to invest in efforts to organize the related communities to re-energize and coordinate their interest in SCI research. These communities need to be able to organize, discuss and prioritize research efforts nationally and internationally, and across the spectrum of corporate, nonprofit and government interests. Some efforts of this kind are underway, both in Europe and in North America, but their scale is small compared to the challenges faced. Nonclassical clinical trial designs such as Bayesian approaches may also reduce the numbers of subjects required to examine efficacy in SCI and this is the topic of a companion manuscript. Such study designs are currently being tested for hypothermia in pediatric brain injury [50].

\section{The role of foundations and other nonprofit organizations}

Nonprofit NGOs related to SCI are diverse in size, organization and resources but they can all benefit from sharing best practices and increased coordination. There are notable examples within the community, including the ICCP, formed in 1997, and continued and expanded in 2006 by formation of an academic-industry-foundation partnership, SCOPE (http://scope-sci.org).

In addition to academic and industry sponsors, NGOs can contribute to clinical trial planning, recruitment and retention efforts. According to a 2017 on-line survey of clinical trial participation from a patient perspective, lack of awareness is a significant reason $(61 \%)$ for not participating [51]. An on-line survey reported individuals with amyotrophic lateral sclerosis, Parkinson's Disease or multiple sclerosis have been participating in clinical trials at higher rates when they were part of a registry [51]. In the context of congenital or developmental diseases, registries of individuals with a rare disorder can serve important recruitment and communication functions. This approach has been less readily applied to acute neurotrauma, but some nonprofit SCI organizations are now investigating such options.

A complementary approach is to directly inform people of a trial for which they could be eligible and instruct them to contact the trial organizer directly if they choose. Eight SCIfocused NGOs have agreed to collaborate to support the development of a new interactive website to do this as part of a new international endeavor called the SCI Trials Toolbox (SCITT). Transparent curation of clinical trial listings will allow presentation of consistent and relevant information that will help potential participants, as well as clinicians to better understand individual trials. People can then directly contact study investigators about their participation. Significant improvements to recruitment are expected when people living with SCI can read lay-language descriptions of SCI studies and review the inclusion/exclusion criteria. This effort arose from 2 independent meetings in 2016, PRAXIS (http://www.rickhanseninstitute.org/images/doc/Praxis2016_ Conference_Report_November2016.pdf) in Vancouver, Canada, and a Translational SCI-NGO Meeting in Ittingen, Switzerland which identified top priorities for successful translational research, including improved recruitment strategies. These meetings launched a number of continuing efforts, one of which led to this paper.

\section{Limitations of the review}

This consideration of recruitment issues relied on the experience and conclusions of its individual authors and arose from conference discussion of problems facing the field. The conclusions are therefore weighted by experience in North America and Europe and do not cover in any depth the issues faced in other parts of the world, where conditions can be quite different, based on populations and the medical systems in place. Much more could be done to develop these themes with respect to the international potential for expanded trial networks. Another weakness was engendered by the frequency with which trials of the past have gone unpublished or incompletely published, so that lessons that could have been learned are lost to the field.

\section{Conclusions}

Recent trials have found it difficult to reproduce the rates of enrollment and retention experienced in larger studies of past decades. Improvements in clinical trial design, sensitive, accurate outcome measures, and quantifiable surrogate markers may all help to reduce the numbers required for effective studies. Increased cooperation between the larger nonprofit SCI groups and improved integration of NGO and government funding may help improve the recruitment environment. More can be done to bring together consumer and provider groups to enhance overall coordination and productivity.

Funding Funding was provided by American Spinal Injury Association (ASIA), Christopher \& Dana Reeve Foundation, (CDRF) Craig H. Neilsen Foundation (CHN), International Foundation for Research in Paraplegia (IRP), International Spinal Research Trust (ISRT), Rick Hansen Institute (RHI), Spinal Cord Outcomes Partnership Endeavor (SCOPE), and Wings for Life (WfL).

Author contributions $\mathrm{ARB}$ and $\mathrm{JH}$ were responsible for manuscript assembly and also contributed individually to the paper. All remaining authors (AC, JWF, JDG, NK, SNK, BKK, DPL, NW, and JDS) contributed individually to the paper. 


\section{Compliance with ethical standards}

Conflict of interest The authors declare that they have no conflict of interest.

Publisher's note: Springer Nature remains neutral with regard to jurisdictional claims in published maps and institutional affiliations.

\section{References}

1. Fawcett JW, Curt A, Steeves JD, Coleman WP, Tuszynski MH, Lammertse D, et al. Guidelines for the conduct of clinical trials for spinal cord injury as developed by the ICCP panel: spontaneous recovery after spinal cord injury and statistical power needed for therapeutic clinical trials. Spinal Cord. 2007;45:190-205.

2. Steeves JD, Lammertse D, Curt A, Fawcett JW, Tuszynski MH, Ditunno J, et al. Guidelines for the conduct of clinical trials for spinal cord injury as developed by the ICCP panel: clinical trial outcome measures. Spinal Cord. 2007;45:206-21.

3. Tuszynski MH, Steeves JD, Fawcett JW, Lammertse D, Kalichman M, Rask C, et al. Guidelines for the conduct of clinical trials for spinal cord injury as developed by the ICCP panel: clinical trial inclusion/exclusion criteria and ethics. Spinal Cord. 2007;45:222-31

4. Lammertse D, Tuszynski MH, Steeves JD, Curt A, Fawcett JW, Rask C, et al. Guidelines for the conduct of clinical trials for spinal cord injury as developed by the ICCP panel: clinical trial design. Spinal Cord. 2007;45:232-42.

5. Singh A, Tetreault L, Kalsi-Ryan S, Nouri A, Fehlings MG. Global prevalence and incidence of traumatic spinal cord injury. Clin Epidemiol. 2014;6:309-31.

6. Lee BB, Cripps RA, Fitzharris M, Wing PC. The global map for traumatic spinal cord injury epidemiology: update 2011, global incidence rate. Spinal Cord. 2014;52:110-6.

7. Lee RS, Noonan VK, Batke J, Ghag A, Paquette SJ, Boyd MC, et al. Feasibility of patient recruitment into clinical trials of experimental treatments for acute spinal cord injury. J Clin Neurosci. 2012;19:1338-43.

8. Thibault-Halman G, Rivers CS, Bailey CS, Tsai EC, Drew B, Noonan VK, et al. Predicting recruitment feasibility for acute spinal cord injury Clinical Trials in Canada Using National Registry Data. J Neurotrauma. 2017;34:599-606.

9. Minei JP, Fabian TC, Guffey DM, Newgard CD, Bulger EM, Brasel KJ, et al. Increased trauma center volume is associated with improved survival after severe injury: results of a Resuscitation Outcomes Consortium study. Ann Surg. 2014;260:456-64.

10. Grossman RG, Toups EG, Frankowski RF, Burau KD, Howley S. North American Clinical Trials Network for the treatment of spinal cord injury: goals and progress. J Neurosurg Spine. 2012;17 (1 Suppl):6-10.

11. Krebs J, Katrin Brust A, Tesini S, Guler M, Mueller G, Velstra I$\mathrm{M}$, et al. Study participation rate of patients with acute spinal cord injury early during rehabilitation. Spinal Cord. 2015;53:738-42.

12. Kwon BK, Ghag A, Dvorak MF, Tetzlaff W, Illes J. Expectations of benefit and tolerance to risk of individuals with spinal cord injury regarding potential participation in clinical trials. J Neurotrauma. 2012;29:2727-37.

13. Dididze M, Green BA, Dietrich WD, Vanni S, Wang MY, Levi AD. Systemic hypothermia in acute cervical spinal cord injury: a case-controlled study. Spinal Cord. 2013;51:395-400.

14. Grossman RG, Fehlings MG, Frankowski RF, Burau KD, Chow DS, Tator C, et al. A prospective, multicenter, phase I matchedcomparison group trial of safety, pharmacokinetics, and preliminary efficacy of riluzole in patients with traumatic spinal cord injury. J Neurotrauma. 2014;31:239-55.

15. Bracken MB, Shepard MJ, Hellenbrand KG, Collins WF, Leo LS, Freeman DF, et al. Methylprednisolone and neurological function 1 year after spinal cord injury: results of the National Acute Spinal Cord Injury Study. J Neurosurg. 1985;63:704-13.

16. Bracken MB, Shepard MJ, Collins WF, Holford TR, Young W, Baskin D, et al. A randomized, controlled trial of methylprednisolone or naloxone in the treatment of acute spinal-cord injury: results of the second national acute spinal cord injury study. $\mathrm{N}$ Engl J Med. 1990;322:1405-11.

17. Bracken MB, Shepard MJ, Collins WF, Holford TR, Baskin D, Eisenberg HM, et al. Methylprednisolone or naloxone treatment after acute spinal cord injury: 1-year follow-up data. J Neurosurg. 1992;76:23-31.

18. Bracken MB, Shepard MJ, Holford TR, Leo-Summers L, Aldrich $\mathrm{EF}, \mathrm{Fazl} \mathrm{M}$, et al. Administration of methylprednisolone for 24 or $48 \mathrm{~h}$ or tirilazad mesylate for $48 \mathrm{~h}$ in the treatment of acute spinal cord injury: Results of the third National Acute Spinal Cord Injury randomized controlled trial. J Am Med Assoc. 1997;277:1597-604.

19. Geisler FH, Dorsey FC, Coleman WP. Recovery of motor function after spinal-cord injury-a randomized, placebo controlled trial with GM-1 ganglioside. N Engl J Med. 1991;324:1829-38.

20. Geisler FH, Coleman WP, Grieco G, Poonian D, the Sygen ${ }^{\circledR}$ Study Group. Recruitment and early treatment in a multicenter study of acute spinal cord injury. Spine. 2001;26:S58-67.

21. Geisler FH, Coleman WP, Grieco G, Poonian D, the Sygen ${ }^{\circledR}$ Study Group. Measurements and recovery patterns in a multicenter study of acute spinal cord injury. Spine. 2001;26:S68-S86.

22. Tadié M, Gaviria M, Mathé J-F, Menthonnex Ph, Loubert G, Lagarrigue J. et al. Early care and treatment with a neuroprotective drug, gacyclidine in patients with acute spinal cord injury. Rachis. 2003;15:363-76.

23. Jones LAT, Lammertse DP, Charlifue SB, Kirshblum SC, Apple DF, Ragnarsson KT, et al. A phase 2 autologous cellular therapy trial in patients with acute, complete spinal cord injury: pragmatics, recruitment, and demographics. Spinal Cord. 2010;48:798-807.

24. Nance PW, Bugaresti J, Shellenberger K, Sheremata W, MartinezArizala A. Efficacy and safety of tizanidine in the treatment of spasticity in patients with spinal cord injury. Neurology. 1994;44 (suppl 9):S44-S52.

25. Cardenas DD, Ditunno J, Graziani V, Lammertse D, Potter P, Sipski M, et al. Phase 2 trial of sustained-release fampridine in chronic spinal cord injury. Spinal Cord. 2007;45:158-68.

26. Cardenas DD, Ditunno JF, Graziani V, McLain AB, Lammertse DP, Potter PJ, et al. Two phase 3, multicenter, randomized, placebocontrolled clinical trials of fampridine-SR for treatment of spasticity in chronic spinal cord injury. Spinal Cord. 2014;52:70-76.

27. Fehlings MG, Nakashima H, Nagoshi N, Chow DS, Grossman RG, Kopjar B. Rationale, design and critical end points for the Riluzole in Acute Spinal Cord Injury Study (RISCIS): a randomized, double-blinded, placebo-controlled parallel multi-center trial. Spinal Cord. 2016;54:8-15.

28. Anderson KD. Targeting recovery: priorities of the spinal cordinjured population. J Neurotrauma. 2004;21:1371-83.

29. Illes J, Reimer JC, Kwon BK. Stem cell clinical trials for spinal cord injury: readiness, reluctance, redefinition. Stem Cell Rev. 2011;7:997-1005.

30. Eijkholt M, Kwon BK, Illes J. Dissociations in the meaning of risk between health-care professionals and individuals with spinal cord injury. Spinal Cord. 2011;51:909-12.

31. Anderson KD, Cowan RE, Horsewell J. Facilitators and Barriers to Spinal Cord Injury Clinical Trial Participation: Multi-National Perspective of People Living with Spinal Cord Injury. J Neurotrauma. 2016;33:493-9. 
32. Levi AD, Anderson KD, Okondwo DO, Park P, Bryce TN, Kurpad SN, et al. Clinical Outcomes from a Multi-Center Study of Human Neural Stem Cell Transplantation in Chronic Cervical SCI. J Neurotrauma. https://doi.org/10.1089/neu.2018.5843.

33. Furlan JC, Sakakibara BM, Miller WC, Krassioukov AV. Global incidence and prevalence of traumatic spinal cord injury. Can J Neurol Sci. 2013;40:456-64.

34. Schuld C, Wiese J, Franz S, Putz C, Stierle I, Smoor I, et al. Effect of formal training in scaling, scoring and classification of the International Standards for Neurological Classification of Spinal Cord Injury. Spinal Cord. 2013;51:282-208.

35. Kucher K, Johns D, Maier D, Abel R, Badke A, Baron H, et al. First-in-man intrathecal application of neurite growth-promoting anti-nogo-a antibodies in acute spinal cord injury. Neurorehabil Neural Repair. 2018;32:578-89.

36. Edwards P, Arango M, Balica L, Cottingham R, El-Sayed H, et al. Final results of MRC CRASH, a randomised placebo-controlled trial of intravenous corticosteroid in adults with head injuryoutcomes at 6 months. Lancet. 2005;365:1957-9.

37. Yang ML, Li JJ, So KF, Chen JY, Cheng WS, Wu J, et al. Efficacy and safety of lithium carbonate treatment of chronic spinal cord injuries: a double-blind, randomized, placebocontrolled clinical trial. Spinal Cord. 2012;50:141-6.

38. Fehlings MG, Nakashima H, Nagoshi N1, Chow DS, Grossman RG, Kopjar B. Rationale, design and critical end points for the Riluzole in Acute Spinal Cord Injury Study (RISCIS): a randomized, double-blinded, placebo-controlled parallel multi-center trial. Spinal Cord. 2016;54:8-15.

39. Kwon BK, Hillyer J, Tetzlaff W. Translational research in spinal cord injury: a survey of opinion from the SCI community. J Neurotrauma. 2009;27:21-33.

40. Kwon BK, Okon EB, Tsai E, Beattie MS, Bresnahan JC, Magnuson DK, et al. A grading system to evaluate objectively the strength of pre-clinical data of acute neuroprotective therapies for clinical translation in spinal cord injury. $\mathrm{J}$ Neurotrauma. 2011;28:1525-43.

41. Kwon BK, Soril LJ, Bacon M, Beattie MS, Blesch A, Bresnahan JC, et al. Demonstrating efficacy in preclinical studies of cellular therapies for spinal cord injury-how much is enough? Exp Neurol. 2013;248:30-44.

42. Curt A, Levi AD, Schwab JM. Challenges to translation and the hippocratic oath by premature termination of spinal cord stem cellbased trials. JAMA Neurol. 2017;74:635-6.

43. Wu X, Liu J, Tanadini LG, Lammertse DP, Blight AR, Kramer $\mathrm{JL}$, et al. Challenges for defining minimal clinically important difference (MCID) after spinal cord injury. Spinal Cord. 2015;53:84-91.

44. Tanadini LG, Steeves JD, Hothorn T, Abel R, Maier D, Schubert $\mathrm{M}$, et al. Identifying homogeneous subgroups in neurological disorders: unbiased recursive partitioning in cervical complete spinal cord injury. Neurorehabil Neural Repair. 2014;28:507-15.

45. Tanadini LG, Hothorn T, Jones LA, Lammertse DP, Abel R, Maier $\mathrm{D}$, et al. Toward inclusive trial protocols in heterogeneous neurological disorders: prediction-based stratification of participants with incomplete cervical spinal cord injury. Neurorehabil Neural Repair. 2015;29:867-77.

46. Seibold H, Zeileis A, Hothorn T. Model-based recursive partitioning for subgroup analyses. Int J Biostat. 2016;12:45-63.

47. Reed R, Mehra M, Kirshblum S, Maier D, Lammertse D, Blight A, et al. Spinal cord ability ruler: an interval scale to measure volitional performance after spinal cord injury. Spinal Cord. 2017;55:730-8.

48. Itzkovich M, Shefler H, Front L, Gur-Pollack R, Elkayam K, Bluvshtein V, et al. SCIM III (Spinal Cord Independence Measure version III): reliability of assessment by interview and comparison with assessment by observation. Spinal Cord. 2018;56:46-51.

49. McDonald AM, Knight RC, Campbell MK, Entwistle VA, Grant $\mathrm{AM}$, Cook JA, et al. What influences recruitment to randomized controlled trials? A review of trials funded by two UK funding agencies. Trials. 2006;7:9.

50. Meurer WJ, Barsan WG. Spinal cord injury neuroprotection and the promise of flexible adaptive clinical trials. World Neurosurg. 2014;82:e541-546.

51. DasMahapatra P, Raja P, Gilbert J, Wicks P. Clinical trials from the patient perspective: survey in an online patient community. BMC Health Serv Res. 2017;17:166-76. 